\title{
Delimitando la agresión adolescente: Estudio diferencial de los patrones de agresión reactiva y proactiva.
}

\author{
Laura López-Romero, Estrella Romero \& Beatriz González-Iglesias. ${ }^{1}$ \\ Universidad de Santiago de Compostela
}

Premio SEIC Jóvenes Investigadores
VIII Congreso de Criminología (San Sebastián, 2011)

RESUMEN

La conducta agresiva constituye un fenómeno complejo en el que se recogen, bajo una misma etiqueta, diversos comportamientos que van a diferir en múltiples aspectos. Dicha diversidad ha llevado a plantear modelos de clasificación entre los que cabe destacar la diferenciación entre agresión de tipo reactivo, considerada como una reacción defensiva ante un acontecimiento determinado, y agresión proactiva, definida por su carácter instrumental, premeditado y ofensivo. Con el fin de analizar los correlatos de la agresión reactiva y proactiva se ha examinado su relación con un conjunto de variables analizadas en una muestra de 138 participantes (12-17 años). Los resultados permitieron observar la presencia de relaciones diferenciales y delimitar el fenómeno de la conducta agresiva adolescente, justificando la diferenciación entre agresión reactiva, relacionada con impulsividad, problemas de internalización o menor competencia a nivel social, y agresión proactiva, caracterizada por menores niveles de ansiedad o neuroticismo, y una mayor presencia de rasgos psicopáticos.

Palabras clave: Agresión reactiva, Agresión proactiva, Adolescencia, Correlatos

\footnotetext{
1 Toda la correspondencia relacionada con este trabajo podrá ser enviada a Laura López Romero. Departamento de Psicología Clínica y Psicobiología. Facultad de Psicología. Campus Vida. 15782, Santiago de Compostela. e-mail: laura.lopez.romero@usc.es. Tfno: 8818-13948.
} 


\begin{abstract}
Delimiting adolescent aggression: A differential study of patterns of reactive and proactive aggression. Aggressive behaviour is a complex phenomenon that covers, under the same label, different behaviours which will differ in many ways. This diversity has led to propose a number of classification models among which it must be underlined the differentiation between reactive aggression, considered a defensive reaction to a particular event, and proactive aggression, defined by its instrumental, deliberate and offensive nature. In order to analyse the correlates of reactive and proactive aggression, this study has examined their relationship with a set of variables analysed in a sample of 138 participants (aged 12 to 17). The results showed a pattern of differential correlations, and allowed to delimit the phenomenon of adolescent aggressive behaviour. These results justify the distinction between reactive aggression, related to impulsivity, internalizing problems and lower social competence level, and proactive aggression, characterized by lower levels of anxiety or neuroticism, and greater presence of psychopathic traits.
\end{abstract}

Keywords: Reactive aggression, Proactive aggression, Adolescence, Correlates 


\section{Introducción}

La agresión hace referencia a un amplio conjunto de comportamientos de naturaleza física, verbal o psicológica, cuya prevalencia se incrementa de forma notable durante la adolescencia, especialmente como parte de conductas antisociales y/o delictivas en las que se sobrepasa el límite de lo socialmente permitido (Hubbard, McAuliffe, Morrow y Romano, 2010; López-Romero y Romero, 2010). Este tipo de comportamientos, generalmente dirigidos a herir o dañar a otros (Berkowitz, 1993), han mostrado en repetidas ocasiones su papel en la infancia como factor de riesgo clave en el desarrollo de problemas de conducta, conducta antisocial adolescente y delincuencia futura (e.g., Barker, Tremblay, Nagin, Vitaro y Lacourse, 2006; Vitaro, Gendreau, Tremblay y Oligny, 1998).

Teniendo en cuenta la heterogeneidad del constructo, se han planteado diversas clasificaciones o tipologías de conducta agresiva con el fin de profundizar en el conocimiento de su etiología, desarrollo y posterior evolución (Raine et al., 2006). De este modo, las diversas manifestaciones englobadas bajo la etiqueta de conducta agresiva han sido analizadas en función de aspectos como su naturaleza o modo de expresión (física/verbal), su dirección (directa/indirecta) o la motivación que las impulsa (reactiva/proactiva; Kokko y Pulkkinen, 2005).

Todas las conceptualizaciones planteadas hasta el momento han permitido ir desglosando un fenómeno multidimensional caracterizado por la heterogeneidad de los comportamientos que lo forman, así como examinar los diversos factores que los provocan y las consecuencias que de ellos se derivan (Cohen, Hsueh, Russell y Ray, 2006). Sin embargo, de todas las clasificaciones propuestas, la que más atención ha recabado hasta el momento ha sido aquélla basada en la motivación que subyace al comportamiento agresivo (Marsee y Frick, 2007); es decir, la distinción planteada entre agresión reactiva y agresión proactiva (Dodge, 1991; Dodge y Coie, 1987). La agresión reactiva, derivada de la Teoría de la Frustración-Agresión, se describe como una respuesta a algún tipo de agresión, amenaza o provocación, real o percibida, a la que suelen acompañar manifestaciones de cólera o ira. Por su parte, la agresión de tipo proactivo se define por su falta de emoción y su carácter instrumental, premeditado y dirigido a la consecución de cualquier tipo de meta o recompensa. 
A pesar de que existen autores que critican y cuestionan este tipo de distinción, especialmente debido al nivel de co-ocurrencia que manifiestan ambas tipologías de conducta agresiva (e.g., Bushman y Anderson, 2001), son muchos los trabajos que han probado la utilidad de esta clasificación defendiendo, en los últimos años, su conceptualización como un continuo dimensional (Hubbard et al., 2010).

Ciertamente, se ha observado una elevada correlación entre ambos tipos de respuestas agresivas, lo cual supone una importante limitación que debe ser explicada a la hora de analizar dicha clasificación (Frick y Marsee, 2006). Del mismo modo, las relaciones observadas entre ambos tipos de comportamientos parecen presentarse de forma asimétrica. En este sentido, se ha observado que existe un importante número de jóvenes que manifiestan únicamente conductas agresivas de tipo reactivo, al tiempo que los jóvenes que manifiestan comportamientos agresivos proactivos suelen mostrar también elevados niveles de agresión reactiva (e.g., Dodge y Coie, 1987; Muñoz, Frick, Kimonis y Aucoin, 2008). De este modo, se ha llegado a plantear la existencia de dos grupos diferenciados de niños y jóvenes agresivos; por una parte estarían aquellos jóvenes con elevados niveles de agresividad y en los que se manifestarían conductas agresivas tanto de tipo reactivo como proactivo; por otra parte, estaría el grupo más numeroso en el que se englobarían los jóvenes que únicamente responden con conductas agresivas de tipo reactivo y en los que los niveles de agresividad suelen ser significativamente menores (Crapanzano, Frick y Terranova, 2010).

Sin embargo, y a pesar del solapamiento observado, no en todos los sujetos se manifiestan de forma conjunta ambos tipos de agresión por lo que han sido muchos los trabajos que han logrado sustentar la distinción realizada entre agresión reactiva y proactiva. Por una parte, los análisis factoriales exploratorios y confirmatorios han constatado la existencia de dos factores bien diferenciados (e.g., Little, Jones, Henrich y Hawley, 2003), siendo las conductas agresivas informadas tanto por profesores y padres como por amigos o por los propios jóvenes (Marsee y Frick, 2007). Por otra parte, se ha observado la existencia de correlatos diferenciales para ambos tipos de conductas agresivas, lo cual supone una pieza clave en el desarrollo de la conceptualización de la agresión reactiva y proactiva. Tal y como se ha planteado en la literatura previa, aun cuando dos medidas están altamente correlacionadas pueden llegar a representar dos 
constructos diferenciados si no presentan el mismo tipo y nivel de relación con otras medidas (Brengen, Vitaro, Tremblay y Lavoie, 2001; Cronbach, 1951).

Por lo tanto, del mismo modo que se debe tener en cuenta el solapamiento existente entre ambos tipos de comportamientos agresivos, es necesario analizar y explicar la existencia de correlatos claramente diferenciados entre uno y otro (Marsee y Frick, 2007). En este sentido, surge la necesidad de examinar si aquellos jóvenes que únicamente manifiestan agresión de tipo reactivo se diferencian significativamente y de forma cualitativa en una serie de variables y correlatos emocionales, cognitivos o conductuales de aquellos sujetos que manifiestan conductas agresivas más severas caracterizadas por respuestas tanto reactivas como proactivas.

Las investigaciones llevadas a cabo hasta el momento han corroborado la existencia de correlatos diferenciales. Por una parte la agresión reactiva se ha relacionado de forma específica, y a nivel cognitivo, con la tendencia a interpretar de forma negativa acciones y situaciones ambiguas atribuyéndoles una connotación hostil que conlleva la percepción de provocación o amenaza, así como con déficits en los procesos de solución de problemas sociales (Dodge y Coie, 1987; Hubbard, Dodge, Cillessen, Coie y Schwartz, 2001). A nivel emocional, la agresión reactiva ha sido relacionada con una elevada impulsividad, bajos niveles de tolerancia a la frustración, mayores niveles de ansiedad y problemas de internalización, y una pobre capacidad de auto-regulación a la hora de responder ante estímulos emocionales (Marsee y Frick, 2007; Vitaro, Brendgen y Tremblay, 2002). Por su parte, la agresión de tipo proactivo se ha relacionado a nivel cognitivo con la tendencia a valorar las respuestas agresivas como una vía útil y efectiva con la que lograr las metas u objetivos anhelados, al tiempo que se relaciona con una baja percepción del castigo como consecuencia posible de la conducta agresiva, es decir, con la tendencia a evaluar de forma positiva la agresión anticipando la obtención de consecuencias positivas y reforzantes (Andreu, Ramírez y Raine, 2006; Dodge, Lochman, Harnish, Bates y Pettit, 1997). Desde un punto de vista afectivo-emocional, la agresión proactiva ha sido relacionada con bajos niveles de reactividad emocional (Hubbard et al., 2002; Muñoz et al., 2008) así como con la manifestación de rasgos de dureza e insensibilidad emocional (baja capacidad para la empatía, ausencia de sentimientos de culpa, pobreza de afecto, etc; Frick et al., 2003), tradicionalmente asociados con el desarrollo de la personalidad psicopática (White y Frick, 2010). 
En el plano conductual, teniendo en cuenta que la manifestación temprana de rasgos de tipo psicopático ha sido relacionada con el desarrollo de problemas de conducta caracterizados por una mayor severidad y persistencia (López-Romero, Romero y Luengo, en prensa), así como las propias características que definen la agresión proactiva, cabe plantear que el empleo de respuestas agresivas de tipo proactivo se enmarcará en patrones de comportamiento problemático y antisocial caracterizados por su mayor severidad y peor pronóstico. En esta línea, diversos trabajos han constatado la relación entre la manifestación temprana de conductas agresivas de tipo proactivo y el desarrollo de conducta externalizante, antisocial y delictiva en la adolescencia y la etapa adulta (Brendgen et al., 2001; Raine et al., 2006; Vitaro et al., 1998).

Finalmente, se han apreciado relaciones diferenciales entre agresión reactiva y proactiva y diversas variables académicas, sociales y familiares. La mayoría de los trabajos apuntan a la existencia de una relación significativa entre agresión de tipo reactivo y mayores problemas a nivel social (especialmente mayor rechazo por parte de los iguales; Hubbard et al., 2010) así como un peor rendimiento académico (Muñoz et al., 2008). Por su parte, la agresión proactiva se ha relacionado con mayor implicación con amigos antisociales, lo cual fomentará el desarrollo de conductas antisociales y delictivas (Brendgen et al., 2001). Del mismo modo, a pesar de que la mayoría de los trabajos no plantean relaciones significativas entre rendimiento académico y agresión proactiva, algunos estudios han constatado su relación con una menor motivación a nivel académico y escolar (e.g., Raine et al., 2006). Desde el punto de vista familiar se ha planteado que ambos tipos de agresión se podrían vincular con experiencias de socialización diferenciales. El empleo de un estilo parental autoritario, relaciones familiares empobrecidas y bajos niveles de implicación y control sobre la conducta de los hijos parecen estar relacionados con la agresión reactiva (Hubbard et al., 2010; Vitaro et al., 1998), mientras que la exposición a modelos agresivos dentro del ambiente familiar podría llegar a favorecer el desarrollo de respuestas agresivas de tipo proactivo como modo de resolver conflictos y conseguir objetivos personales (Vitaro, Brendgen y Barker, 2006).

Teniendo en cuenta los resultados obtenidos hasta el momento, el presente trabajo se plantea con el objetivo de analizar los correlatos que a nivel conductual, emocional, familiar $y$ de personalidad permiten diferenciar entre las conductas agresivas 
adolescentes de tipo reactivo y proactivo, informadas por padres, profesores y los propios jóvenes. De este modo, se pretende justificar la distinción realizada entre agresión reactiva y proactiva como forma de delimitar el fenómeno del comportamiento agresivo en la adolescencia.

\section{Método}

\subsection{Participantes}

Los datos del presente trabajo fueron recogidos en una muestra de 138 jóvenes (68,8\% chicos y $31,2 \%$ chicas) con edades comprendidas entre los 12 y 17 años $(M=$ 13,95; $S x=1,40)$. Todos ellos se encontraban escolarizados en 37 centros de Educación Primaria y Secundaria de la Comunidad Gallega. Atendiendo a la necesidad de contar con múltiples fuentes de información en el estudio del comportamiento infanto-juvenil, dada su variabilidad en función del contexto en que se desarrolla (Molina, 2001), el trabajo se ha elaborado a partir de la información proporcionada por padres (138), profesores (102 tutores académicos) y los propios jóvenes (131).

\subsection{Variables e instrumentos}

A continuación se describen las variables analizadas y los instrumentos empleados. Así mismo, en el Cuadro 1 se puede observar la distribución de instrumentos en función del informante (padres, profesores o niños).

\subsubsection{Agresión reactiva/proactiva}

Parent/Teacher Report of Reactive and Proactive Behaviors (Dodge y Coie, 1987). La valoración de las conductas agresivas de los jóvenes se realizó a través de la versión para padres y profesores de este instrumento, además de una adaptación autoinformada de la versión para padres con el fin de que pudiese ser completada por los propios jóvenes. El cuestionario está formado por 6 ítems, tres de los cuales valoran comportamientos agresivos de tipo reactivo (Agresión reactiva; e.g., "Si alguien me hace algo, normalmente siento que lo hacen a propósito y reacciono enfadándome o peleando") mientras que los tres restantes analizan la manifestación de conductas agresivas de tipo proactivo (Agresión proactiva; e.g., "Amenazo o intimido a otros chicos/as para que las cosas se hagan a mi manera"). Padres, profesores y jóvenes 
debían informar de la frecuencia con la que se producían cada una de las respuestas agresivas descritas en los ítems, en una escala de 1 (Nunca es cierto) a 5 (Casi siempre es cierto).

\subsubsection{Problemas de conducta}

Child Behavioral Checklist (CBCL; Achenbach, 1991a). Este cuestionario valora la presencia de alteraciones conductuales en las dimensiones InteriorizaciónExteriorización, a partir de la información proporcionada por los padres a través de 113 ítems con una escala de respuesta de 0 (No es verdad) a 2 (Muy verdadero o frecuentemente verdad). De las diversas categorías en las que se agrupan los ítems, se seleccionaron las dos dimensiones globales de problemas de conducta: Problemas Externalizantes, formada por 33 ítems que permiten valorar la manifestación de comportamientos agresivos y/o delictivos (e.g., "Es abusón, cruel y desconsiderado con los demás", "Prende fuegos"), y Problemas Internalizantes que, a través de 31 ítems, proporciona una visión global de desajustes a nivel internalizante como elevados niveles ansiedad, síntomas depresivos o abundantes quejas somáticas sin causa física justificada (e.g., "Se siente desgraciado, triste, deprimido").

Teacher's Report Form (TRF; Achenbach, 1991b). Con una estructura similar al CBCL este cuestionario analiza los problemas conductuales informados desde el ámbito académico. De nuevo se seleccionaron las dimensiones globales de Problemas Externalizantes y Problemas Internalizantes, con las que obtener una visión general de diversos tipos de desajuste conductual informados por profesores.

Escala de Conners para padres-Revisada (Conners, 1997). La versión abreviada de este instrumento se compone de 27 ítems en los que los padres deben valorar, en una escala de 0 (Nunca o rara vez) a 3 (Siempre es cierto), la frecuencia con la que observan en sus hijos los comportamientos señalados. Esta escala permite la medición de comportamientos oposicionistas en la infancia, déficits de atención o inquietud motora, así como un índice global de Trastorno por Déficit de Atención e Hiperactividad (TDAH). Con el fin de evitar el solapamiento entre las distintas medidas empleadas en el trabajo, se ha optado por incluir en el estudio únicamente la variable Índice TDAH, formada por 12 de los ítems del cuestionario (e.g., "Tiene poca capacidad para prestar atención", "Se frustra fácilmente cuando se esfuerza"). 
Cuestionario de Conducta Antisocial (CCA; Luengo, Otero, Romero, GómezFraguela y Tavares-Filho, 1999). Este cuestionario permite valorar la implicación de los jóvenes en comportamientos antisociales a través de 37 ítems, con una escala de respuesta de 0 (Nunca) a 4 (Con frecuencia), en los que los jóvenes debían valorar la frecuencia con la que han llevado a cabo conductas relacionadas con el consumo de drogas, la agresión, e vandalismo o el robo ("e.g., plantar fuego a algo: una papelera, una mesa, coche").

\subsubsection{Personalidad psicopática}

Antisocial Process Screening Device (APSD; Frick y Hare, 2001). Esta escala, de la que se ha empleado la versión para padres, profesores y autoinformada, permite valorar la manifestación temprana de rasgos psicopáticos a través de 20 ítems puntuables en una escala de 0 (Totalmente falso) a 2 (Totalmente verdadero). Se ha optado por la estructura inicialmente hallada de dos factores (Frick et al., 1994): Dureza/Insensibilidad emocional (D/IE; e.g., "Tiene emociones superficiales o poco auténticas"), que permite valorar los rasgos afectivos e interpersonales más característicos de la personalidad psicopática, e Impulsividad/Problemas de conducta (I/PC), que permite analizar los aspectos más conductuales del constructo (e.g., "Actúa sin pensar en las consecuencias").

Sub-escala de Búsqueda de Emoción y Aventura (Escala de Búsqueda de Sensaciones para niños; Russo et al., 1993). Esta escala permite obtener una medida de las preferencias de los jóvenes por situaciones novedosas $y$, en ocasiones, peligrosas. Los participantes debían elegir entre 26 pares de afirmaciones aquella con la que estaban más de acuerdo o que mejor los definía (e.g., "Me gustaría escalar una montaña" vs. "Creo que la gente que hace cosas peligrosas como escalar montañas está loca"). La puntuación obtenida mediante este instrumento ha permitido crear una medida global de Búsqueda de sensaciones a partir de la cual valorar en la adolescencia la ausencia de miedo así como la tendencia a buscar nuevas sensaciones, emociones y aventuras, aspectos tradicionalmente relacionados con los problemas de conducta o la personalidad psicopática.

Sub-escala de Impulsividad del I6 (Eysenk, Easting y Pearson, 1984; adaptación española de Silva, Martorell y Clemente, 1986). Para el presente trabajo se empleó una 
versión reducida de esta escala, formada por 12 ítems en forma de pregunta (e.g., “¿Dices y haces cosas sin pensarlas?”) con un formato de respuesta dicotómico ( $\mathrm{Si} / \mathrm{No})$. La puntuación global permitió la creación de la escala Impulsividad a través de la cual se valoró la capacidad de los participantes para pensar antes de actuar, controlar sus impulsos o la tolerancia a la frustración.

Empathy Scale for Children (Bryant, 1982). Con el fin de obtener una medida global de la capacidad que poseen los jóvenes para situarse en el lugar de los demás y comprender sus sentimientos y emociones (i.e., Empatía), se empleó una versión reducida de la escala compuesta por 10 ítems con un formato de respuesta Verdadero/Falso (e.g., "Cuando veo a alguien que está triste me pongo triste yo también"). Dichos ítems se consideran representativos de las tres dimensiones tradicionalmente evaluadas en el análisis de la empatía: Sentimientos de tristeza, Comprensión de sentimientos y Reacción al llanto.

\section{Cuadro 1}

Variables e instrumentos

\begin{tabular}{|c|c|c|c|}
\hline & PADRES & Profesores & Niños \\
\hline $\begin{array}{l}\text { CONDUCTA AGRESIVA } \\
\text { (Reactiva/Proactiva) }\end{array}$ & $\begin{array}{l}\text { - Parent Report of } \\
\text { Reactive and } \\
\text { Proactive } \\
\text { Behaviors (Dodge } \\
\text { y Coie, 1987) }\end{array}$ & $\begin{array}{l}\text { Teacher Report of } \\
\text { Reactive and } \\
\text { Proactive } \\
\text { Behaviors (Dodge } \\
\text { y Coie, 1987) }\end{array}$ & $\begin{array}{l}\text { - Versión adaptada } \\
\text { autoinformada de } \\
\text { la Parent Report of } \\
\text { Reactive and } \\
\text { Proactive } \\
\text { Behaviors (Dodge } \\
\text { y Coie, 1987) } \\
\end{array}$ \\
\hline $\begin{array}{l}\text { PROBLEMAS DE } \\
\text { CONDUCTA }\end{array}$ & $\begin{array}{l}\text { - Child Behavioral } \\
\text { Checklist (CBCL; } \\
\text { Achenbach, } \\
\text { 1991a) } \\
\text { - Escala de Conners } \\
\text { para padres- } \\
\text { Revisada } \\
\text { (Conners, 1997) } \\
\end{array}$ & $\begin{array}{l}\text { Teacher's Report } \\
\text { Form (TRF; } \\
\text { Achenbach, } \\
\text { 1991b) }\end{array}$ & $\begin{array}{l}\text { - Cuestionario de } \\
\text { Conducta } \\
\text { Antisocial (CCA; } \\
\text { Luengo et al., } \\
\text { 1999) }\end{array}$ \\
\hline $\begin{array}{l}\text { PERSONALIDAD } \\
\text { PSICOPÁTICA }\end{array}$ & $\begin{array}{l}\text { - Antisocial Process } \\
\text { Screening Device } \\
\text { (APSD; Frick y } \\
\text { Hare, 2001) }\end{array}$ & $\begin{array}{l}\text { - Antisocial Process } \\
\text { Screening Device } \\
\text { (APSD; Frick y } \\
\text { Hare, 2001) }\end{array}$ & $\begin{array}{l}\text { - Antisocial Process } \\
\text { Screening Device } \\
\text { (APSD; Frick y } \\
\text { Hare, 2001) }\end{array}$ \\
\hline
\end{tabular}




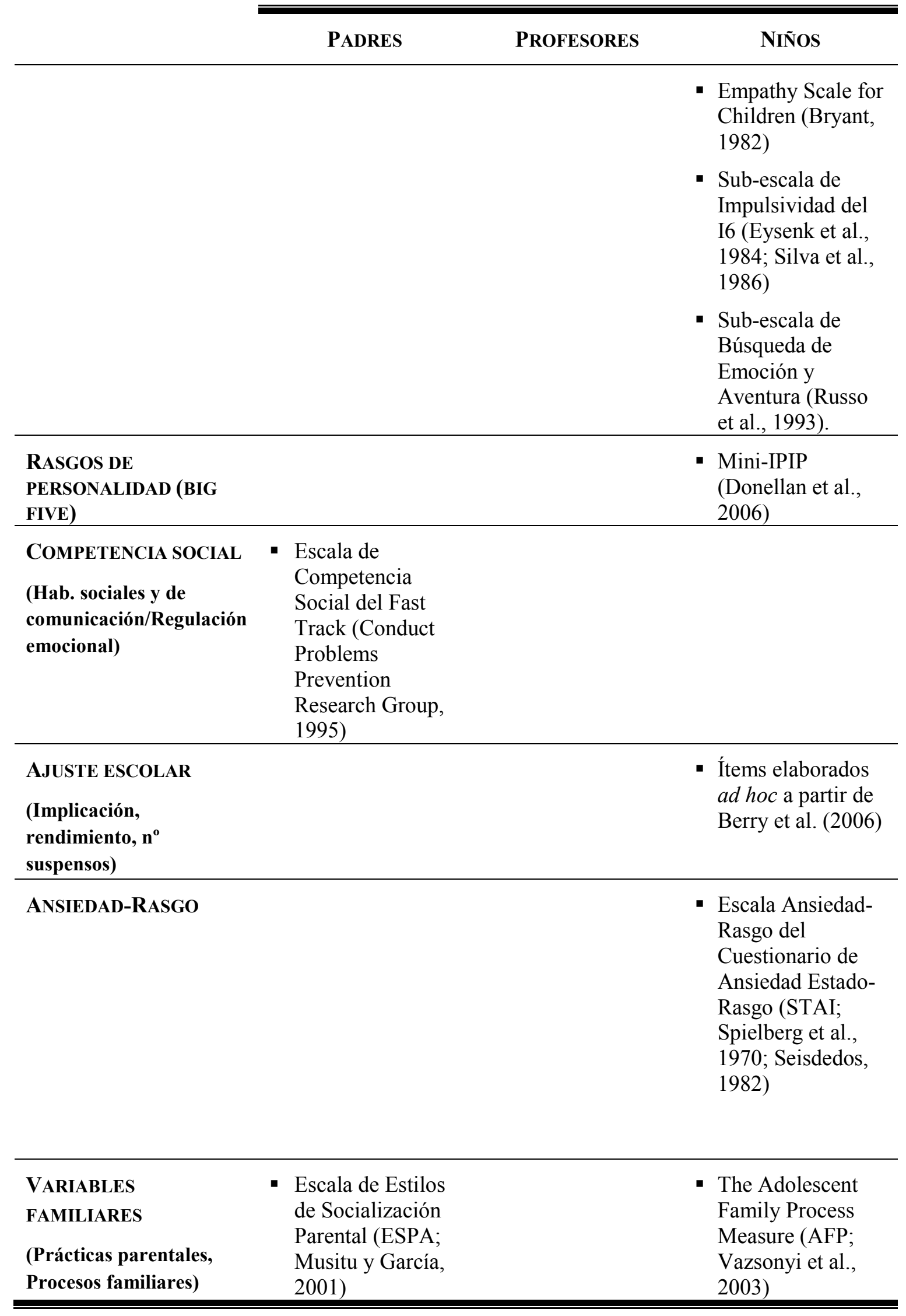




\subsubsection{Rasgos de personalidad (Big Five)}

Mini-IPIP (Donellan, Oswald, Baird y Lucas, 2006). Este cuestionario constituye una versión reducida del International Personality Ítem Pool (IPIP; Goldberg, 1999). A través de 21 ítems que deben ser respondidos en una escala de 0 (Totalmente en desacuerdo) a 4 (Totalmente de acuerdo), proporciona una medida fiable de los rasgos de personalidad tradicionalmente medidos en el Modelo de los Cinco Grandes: Extraversión (e.g., "En las fiestas habla con mucha gente diferente"), Amabilidad (e.g., "No le interesan los problemas de los demás"), Responsabilidad (e.g., "Hace las tareas cotidianas rápido y bien"), Neuroticismo (e.g., "Tiene frecuentes cambios de humor”), e Intelecto o Apertura (e.g., "No le interesan las ideas abstractas").

\subsubsection{Competencia social}

Escala de Competencia Social del Fast Track (Conduct Problems Prevention Research Group, 1991). A partir de los 12 ítems que conforman esta escala los padres proporcionan información acerca de la competencia que a nivel social manifiestan sus hijos. De forma específica, seis de los ítems que conforman la escala permiten valorar el nivel de Habilidades sociales y comunicativas de los adolescentes (e.g., "Escucha los puntos de vista de los demás"). Los seis ítems restantes valoran el grado de Regulación emocional que manifiestan los jóvenes (e.g., "Se enfrenta bien al rechazo"). La tarea de los padres consistía en informar del grado en que se daban cada una de las afirmaciones en una escala de 5 puntos que iba desde 0 (En absoluto) a 4 (Totalmente).

\subsubsection{Ajuste escolar}

Escala de Ajuste Escolar (Ítems elaborados ad hoc a partir de Berry, Phinney, Sam y Vedder, 2006). Con el fin de valorar el grado de ajuste en el ámbito escolar así como rendimiento académico informado por los propios adolescentes, se elaboró una breve escala dividida en dos partes. En primer lugar, 6 ítems destinados a valorar el grado de ajuste e Implicación escolar (e.g., "Por las mañanas me disgusta tener que ir a clase") en una escala de 0 (Muy en desacuerdo) a 4 (Muy de acuerdo). En segundo lugar se elaboraron 4 preguntas destinadas a analizar el nivel de Rendimiento académico ("Crees que tu profesor/a piensa que tu rendimiento escolar es...”), que debían valorar en una 
escala de 5 puntos (Muy malo a Muy bueno); el grado de Absentismo escolar (“¿Has faltado a clase sin causa justificada durante el último mes?"), en la que los jóvenes debían señalar el número de ocasiones en que lo habían realizado (desde Nunca hasta 5 veces o más); y el Número de suspensos (“¿Cuántas asignaturas necesitaste recuperar el curso pasado?"), que permite obtener una nueva medida del rendimiento académico valorándolo en una escala de seis puntos (desde Ninguna hasta Más de 4).

\subsubsection{Ansiedad-rasgo}

Escala Ansiedad-Rasgo del Cuestionario de Ansiedad Estado-Rasgo (STAI; Spielberg, Gorshu y Lushore, 1970; adaptación española de Seisdedos, 1982). Mediante esta escala, se valora la propensión a la ansiedad que, de forma relativamente estable, manifiestan los jóvenes analizando de este modo su tendencia a valorar las situaciones como amenazantes. La escala se compone de 20 ítems (e.g., "Me vienen a la cabeza pensamientos sin importancia y me molestan"), en los que los participantes debían señalar el grado de frecuencia con que se producían las diversas situaciones (Casi nunca, Algunas veces o A menudo).

\subsubsection{Variables familiares}

Escala de Estilos de Socialización Parental (ESPA; Musitu y García, 2001). Esta escala permite obtener información sobre las prácticas parentales empleadas en el manejo de los hijos y los estilos de socialización subyacentes. La información proporcionada por los padres permitió la creación de siete escalas que se corresponden con siete tipos de prácticas disciplinarias que se pueden llegar a emplear en las 29 situaciones que se les plantean a los padres: Afecto ("Le muestro afecto"), Indiferencia ("Me muestro indiferente"), Displicencia ("Me da igual”), Coerción verbal ("Le riño"), Coerción física ("Le pego"), Privación ("Le privo de algo"), y Diálogo ("Hablo con el"). A partir de estas escalas y siguiendo los criterios de los autores, se creó la dimensión de Aceptación/Implicación, a partir de la suma de las escalas Afecto y Diálogo a las que se les restan las de Indiferencia y Displicencia, por saturar de forma negativa en dicha variable, y la dimensión de Coerción/Imposición, en la que se agruparon las escalas Coerción verbal, Coerción física y Privación. De este modo se 
obtienen dos medidas globales del tipo de prácticas que, a nivel general, suelen emplear los padres en el manejo de los hijos.

The Adolescent Family Process Measure (AFP; Vazsonyi, Hibbert y Snider, 2003). Con el fin de obtener una valoración por parte de los jóvenes de los procesos que habitualmente se producen en el ámbito familiar se ha empleado este cuestionario. Compuesto por dos secciones, la primera de ellas permite valorar, en una escala de 0 (Muy en desacuerdo) a 4 (Totalmente de acuerdo), aspectos como la Calidad del Vínculo (5 ítems; e.g., "Mi padre/madre confía en mi”), el nivel de Apoyo (4 ítems; e.g., "A veces mi padre/madre me hace sentir que no estoy a la altura de sus expectativas"), y el grado de Control sobre la conducta del hijo (4 ítems; "En mi tiempo libre fuera de casa mi padre/madre sabe donde estoy y con quien"). En la segunda parte del cuestionario los ítems adquieren forma de pregunta que los jóvenes han de valorar en una escala cuatro puntos (Nunca a Muy a menudo). Dichas preguntas permiten analizar la calidad de la Comunicación familiar (5 ítems; e.g., “Con qué frecuencia hablas con tu padre/madre sobre problemas que tienes en el colegio?"), el nivel de Conflicto (3 ítems; e.g., "¿Con qué frecuencia tienes desacuerdos o discusiones con tu padre/madre?”), y el grado de aprobación de los Amigos (3 ítems; e.g., “¿Con qué frecuencia tu padre/madre aprueba a tus amigos?").

\subsection{Procedimiento}

El estudio se inició con un contacto inicial en el que se detallaron los objetivos del trabajo tanto a las familias como a los responsables de los centros educativos en los que estaban escolarizados los jóvenes. Una vez obtenida la autorización para realizar el estudio, los cuestionarios, administrados por psicólogos cualificados y entrenados en la aplicación de los instrumentos, fueron completados por padres, profesores (preferiblemente los tutores académicos) y los propios jóvenes en los centros educativos. Todo el proceso de evaluación fue llevado a cabo bajo condiciones de garantizada confidencialidad.

\subsection{Análisis estadísticos}

Se han realizado análisis de correlación simple con el fin de examinar la relación existente entre agresión reactiva/proactiva y las diversas variables analizadas. Dado el 
solapamiento tradicionalmente observado entre ambos tipos de agresión, y atendiendo a las recomendaciones realizadas desde la literatura previa (Muñoz et al., 2008), se han realizado también análisis de correlación parcial en los que se ha controlado el efecto de la agresión reactiva o proactiva, según correspondiese.

\section{Resultados}

A continuación se presentan los resultados de los análisis de correlación llevados a cabo entre las variables de agresión reactiva y proactiva informadas por padres, profesores y jóvenes, y diversas variables conductuales, emocionales, familiares y de personalidad. Teniendo en cuenta la relación existente entre agresión reactiva y proactiva $(.65, .63$ y $.56 ; \mathrm{p}<.001$, para padres, profesores y niños respectivamente), se han repetido los análisis parcializando el efecto de la agresión proactiva al examinar los correlatos de la agresión reactiva y viceversa. Los resultados de dichos análisis se presentan entre paréntesis.

En primer lugar, en la Tabla 1 se presentan los resultados hallados en relación a las variables informadas por los padres. Dichos resultados muestran la existencia de relaciones estadísticamente significativas entre ambos tipos de agresión y problemas de conducta, tanto externalizante como internalizante, así como con TDAH, cuya relación con agresión proactiva pierde significación estadística una vez controlada la agresión reactiva. En cuanto a la manifestación temprana de rasgos psicopáticos, las relaciones más intensas se observan con la agresión de tipo proactivo, especialmente una vez parcializado el efecto de la reactiva. Por el contrario, las habilidades sociales y de comunicación así como las de regulación emocional presentan relaciones significativas, e inversas, con ambos tipos de agresión que únicamente se mantienen para la agresión reactiva tras controlar el efecto de la proactiva. Con relación a las prácticas educativas parentales parece que las prácticas coercitivas (castigos físicos o verbales, privación) únicamente se relacionan con las respuestas agresivas de tipo reactivo, tanto en los análisis de correlación simple como en los de correlación parcial. 


\section{Tabla 1}

Correlatos de la agresión reactiva y proactiva informada por los padres

\begin{tabular}{|c|c|c|}
\hline & REACTIVA & Proactiva \\
\hline \multicolumn{3}{|l|}{ Problemas de conducta } \\
\hline CBCL-Externalizante & $.67 * * *(.46 * * *)$ & $.62 * * *(.32 * * *)$ \\
\hline CBCL-Internalizante & $.38 * * *(.18 *)$ & $.39 * * *(.20 *)$ \\
\hline Conners-TDAH & $.57 * * *(.49 * * *)$ & $.34 * * *(-.05)$ \\
\hline \multicolumn{3}{|l|}{ Personalidad psicopática } \\
\hline APSD-D/Ie & $.21 *(.14)$ & $.14(.22 * *)$ \\
\hline APSD-I/Pc & $.25 * *(.07)$ & $.31 * * *(.20 *)$ \\
\hline \multicolumn{3}{|l|}{ Competencia Social } \\
\hline Habilidades sociales y comunicativas & $-.36 * * *(-.24 * *)$ & $-.29 * * *(-.08)$ \\
\hline Habilidades de regulación emocional & $-.48 * * *(-.36 * * *)$ &.$- .32 * * *(-.02)$ \\
\hline \multicolumn{3}{|l|}{ Prácticas parentales- ESPA } \\
\hline Aceptación/Implicación & $-.15(-.18)$ & $-.02(.12)$ \\
\hline Coerción/Imposición & $.31 * * *(.29 * * *)$ & $.15(-.10)$ \\
\hline
\end{tabular}

$* \mathrm{p}<.05 \quad * * \mathrm{p}<.01 \quad * * * \mathrm{p}<.001$

Entre paréntesis se presentan los resultados de las correlaciones parciales

En la Tabla 2 se presentan los resultados de los análisis realizados con la información proporcionada por los profesores desde el ámbito académico. En ella se puede observar, de nuevo, una importante relación entre ambos tipos de agresión y problemas de conducta externalizante. Los desajustes internalizantes, por su parte, se relacionan de forma directa e intensa con agresión reactiva, mientras que la relación con agresión proactiva, en este caso inversa, surge únicamente tras controlar el efecto de las conductas agresivas de tipo reactivo. De este modo, y al contrario de lo observado en la Tabla 1, los mayores niveles de problemas internalizantes se darían entre los jóvenes con mayores niveles de agresión reactiva, al tiempo que dichos problemas se reducirían entre el grupo de jóvenes con tendencia a responder con conductas agresivas proactivas. En cuanto a las variables de personalidad psicopática se observa la existencia de relaciones significativas entre los rasgos de Impulsividad y Problemas de Conducta y ambos tipos de respuestas agresivas. Con relación a la Dureza e insensibilidad 
emocional las relaciones estadísticamente significativas únicamente se mantienen con la agresión reactiva una vez controlado el efecto de la proactiva.

\section{Tabla 2}

Correlatos de la agresión reactiva y proactiva informada por profesores

\begin{tabular}{|c|c|c|}
\hline & REACTIVA & ProActiva \\
\hline \multicolumn{3}{|c|}{ Problemas de conducta } \\
\hline TRF-Externalizante & $.79 * * *\left(.65^{* * *}\right)$ & $.73 * * *(.52 * * *)$ \\
\hline TRF-Internalizante & $.60 * * *(.65 * * *)$ & $.18(-.33 * * *)$ \\
\hline \multicolumn{3}{|c|}{ Personalidad psicopática } \\
\hline APSD-D/Ie & $.61 * * *(.46 * * *)$ & $.41 * * *(.10)$ \\
\hline APSD-I/Pc & $.75^{* * *}(.57 * * *)$ & $.72 * * *(.50 * * *)$ \\
\hline
\end{tabular}

Entre paréntesis se presentan los resultados de las correlaciones parciales

Finalmente, en la Tabla 3 se presentan los resultados de los análisis de correlación realizados entre las variables autoinformadas por los propios jóvenes. Los datos muestran que de nuevo la manifestación de ambos tipos de agresión se relaciona con conducta antisocial y consumo de drogas en la adolescencia. Sin embargo, las relaciones con consumo de drogas pierden significación estadística en las correlaciones parciales. A nivel académico los mayores problemas se observan entre los jóvenes con mayores niveles de agresión proactiva, incluso tras controlar el efecto de la reactiva. De este modo, este grupo de jóvenes mostrarán menores niveles de implicación y rendimiento académico así como mayores niveles de absentismo escolar. Con respecto a la personalidad psicopática, a pesar de que en los análisis iniciales se observan relaciones significativas con ambos tipos de agresión, una vez controlado el efecto de las variables únicamente la búsqueda de sensaciones mantiene dichas relaciones aunque con menor intensidad y significación estadística, al tiempo que los rasgos relacionados con la impulsividad y la conducta problemática mantienen su nivel de relación con la agresión de tipo reactivo. En cuanto a los rasgos de personalidad analizados, Extraversión y Responsabilidad se relacionan tanto con agresión reactiva como con proactiva. Sin embargo, tras parcializar su efecto en los análisis de correlación, 
únicamente se mantienen las relaciones entre extraversión y agresión proactiva y responsabilidad y agresión reactiva, en este último caso de forma inversa. Por otra parte, los mayores niveles de neuroticismo y ansiedad se observan en el grupo de jóvenes con mayores niveles de agresión de tipo reactivo. Por último, las variables familiares informadas por los jóvenes se relacionan significativamente con la conducta agresiva reactiva una vez controlada la proactiva; de este modo, los jóvenes con mayores niveles de agresión reactiva informan de procesos familiares disfuncionales caracterizados especialmente por un escaso control, una bajo nivel de apoyo y la presencia de relaciones familiares conflictivas. 
Tabla 3. Correlatos de la agresión reactiva y proactiva informada por los jóvenes

\begin{tabular}{|c|c|c|}
\hline & REACTIVA & ProActiva \\
\hline \multicolumn{3}{|l|}{ Problemas de conducta } \\
\hline CCA-Conducta antisocial & $.54 * * *(.35 * * *)$ & $.49 * * *(.27 * *)$ \\
\hline CCA-Consumo drogas & $.25 * *(.17)$ & $.18 *(.08)$ \\
\hline \multicolumn{3}{|l|}{ Ajuste escolar } \\
\hline Implicación & $-.36 * * *(-.20 *)$ & $-.39 * * *(-.25 * *)$ \\
\hline Rendimiento & $-.30 * * *(-.12)$ & $-.36 * * *(-.26 * *)$ \\
\hline Absentismo & $.24 * *(.05)$ & $.40 * * *(.33 * * *)$ \\
\hline $\mathrm{N}^{\mathrm{o}}$ suspensos & $.22 * *(.09)$ & $.23 * *(-.17)$ \\
\hline \multicolumn{3}{|l|}{ Personalidad psicopática } \\
\hline APSD-D/Ie & $.23 * *(.12)$ & $.23 * * *(.14)$ \\
\hline APSD-I/Pc & $.30 * * *(.48 * * * *)$ & $.46 * * *(.16)$ \\
\hline Búsqueda de sensaciones & $.36 * * *(-.22 *)$ & $.34 * * *(.18 *)$ \\
\hline Impulsividad & $.44^{* * * *}(.31 * * *)$ & $.37 * * *(.17 *)$ \\
\hline Empatía & $-.11(-.08)$ & $-.11(-.05)$ \\
\hline \multicolumn{3}{|c|}{ Rasgos de personalidad (Big Five) } \\
\hline Extraversión & $.27 * *(.11)$ & $.30 * * *(.19 *)$ \\
\hline Afabilidad & $-.05(.04)$ & $-.14(-.14)$ \\
\hline Responsabilidad & $-.36 * * *(-.26 * *)$ & $-.32 * * *(-.15)$ \\
\hline Neuroticismo & $.29 * * *(.30 * * *)$ & $.08(-.12)$ \\
\hline Intelecto & $-.06(.01)$ & $-.13(-.12)$ \\
\hline \multicolumn{3}{|l|}{ Ansiedad } \\
\hline Ansiedad-rasgo & $.17 *(.21 *)$ & $.01(-.11)$ \\
\hline \multicolumn{3}{|l|}{ Procesos familiares (AFP) } \\
\hline Vínculo & $-.20 *(-.11)$ & $-.21 *(-.12)$ \\
\hline Control & $-.31 * * *(-.20 *)$ & $-.28 * * *(-.11)$ \\
\hline Comunicación & $-.24 * *(-.12)$ & $-.28 * * *(-.15)$ \\
\hline Conflicto & $.36 * * *(.34 * * *)$ & $.14(-.03)$ \\
\hline Ароуо & $-.28 * * *(-.21 *)$ & $-.21 *(.01)$ \\
\hline Amigos & $-.01(-.02)$ & $.01(.02)$ \\
\hline
\end{tabular}

$* \mathrm{p}<.05 \quad * * \mathrm{p}<.01 \quad * * * \mathrm{p}<.001$

Entre paréntesis se presentan los resultados de las correlaciones parciales 


\section{Discusión}

Con el presente trabajo se pretendía analizar la existencia de correlatos diferenciales entre los dos tipos de agresión que mayor interés han suscitado en la literatura previa: agresión reactiva vs. agresión proactiva. Ambos tipos de respuestas agresivas constituyen una conceptualización de la agresión adolescente basada en la motivación que subyace a la conducta adolescente (Kokko y Pulkinen, 2005). Dicha conceptualización responde a la necesidad de profundizar en un fenómeno, la conducta agresiva adolescente, caracterizado por la heterogeneidad de los comportamientos que lo conforman, facilitando así la comprensión de su etiología y posterior evolución (Raine et al., 2006; Walters, 2005).

A pesar de que son muchos los autores que han cuestionado esta clasificación debido al evidente solapamiento entre agresión reactiva y proactiva (Bushman y Anderson, 2001), han sido múltiples y variados los trabajos que han constatado la utilidad del constructo (Hubbard et al., 2010). Una gran mayoría de estos trabajos ha centrado su interés en la detección de relaciones diferenciadas entre agresión reactiva y proactiva, y un amplio conjunto de variables que abarcan desde aspectos cognitivoemocionales, como respuestas a nivel conductual o factores sociales como el papel de la familia o la relación con los iguales (Muñoz et al., 2008). Es precisamente la existencia de correlatos diferenciales en cuanto al tipo y nivel de relación con otras variables la que permite plantear la existencia de dos constructos diferenciados (Crapanzano et al., 2010; Cronbach, 1951) con sus particulares factores etiológicos, respuestas conductuales y trayectorias evolutivas (Barker et al., 2006; Raine et al., 2006).

Los resultados presentados en el apartado anterior corroboran en buena medida los observados en investigaciones anteriores. Sin embargo, es necesario tener en cuenta el solapamiento existente entre ambos tipos de agresión a la hora de realizar las interpretaciones pertinentes (Frick y Marsee, 2006). Es por ello que todos los análisis fueron repetidos parcializando el efecto de la agresión reactiva o proactiva según correspondiese. Dado que el solapamiento entre dichas variables supone una importante limitación en el estudio de la agresión adolescente, las conclusiones obtenidas en el trabajo se centrarán en los resultados obtenidos a través de las correlaciones parciales, una vez controlado el solapamiento anteriormente referido (Marsee y Frick, 2007). 
En primer lugar, se ha observado que tanto la agresión reactiva como la proactiva se relacionan con problemas de conducta, informados por padres y profesores, y con conducta antisocial autoinformada por los propios adolescentes. A pesar de que la mayoría de los trabajos asocian la agresión proactiva con problemas de conducta severos, conducta antisocial y delincuencia futura (Brendgen et al., 2001; Raine et al., 2006; Vitaro et al., 2006), cabe pensar que, desde una perspectiva transversal, ambos tipos de agresión se relacionen con conductas problemáticas cuya prevalencia tiende a incrementarse de forma notable en la etapa adolescente (López-Romero et al., 2010). Por otra parte, los instrumentos empleados en el análisis de problemas de conducta (CBCL, TRF y CCA) recogen ítems destinados a valorar específicamente las conductas agresivas; en este sentido, es probable que aquellos participantes que manifiesten comportamientos agresivos, sean del tipo que sean, obtengan también puntuaciones elevadas en los cuestionarios empleados. Con el fin de profundizar en la relación existente entre agresión reactiva-proactiva y el desarrollo de problemas de conducta será necesario plantear nuevos diseños que permitan corroborar, desde una perspectiva longitudinal, si la manifestación de conductas agresivas a edades tempranas supone un factor de riesgo relevante para el desarrollo de conductas antisociales y/o delictivas en el futuro (Vitaro et al., 1998). Por otra parte, la valoración de TDAH únicamente se relaciona con conductas agresivas de tipo reactivo, tradicionalmente asociadas con impulsividad y déficits de atención (e.g., Raine et al., 2006). Por el contrario, los peores niveles de ajuste escolar se observan entre los jóvenes que manifiestan mayores niveles de agresión proactiva. A pesar de que son escasos los trabajos que relacionan la agresión proactiva con rendimiento académico, estudios longitudinales como el realizado por Raine et al., (2006) pusieron de manifiesto que entre los jóvenes con mayor agresión proactiva se observaban los menores niveles de motivación académica, lo cual se traduciría en una menor implicación, mayores niveles de absentismo escolar $\mathrm{y}$, por consiguiente, un peor rendimiento (e.g., mayor número de suspensos).

Relacionado también con la conducta problema se ha observado que, según la información proporcionada por los padres, los problemas de conducta internalizantes se relacionan con ambos tipos de conducta agresiva. Sin embargo, desde el punto de vista de los profesores y los propios jóvenes, únicamente la agresión reactiva se relaciona con elevados niveles de ansiedad, neuroticismo y otros problemas de tipo internalizante, 
corroborando los hallazgos obtenidos hasta el momento en investigaciones previas (e.g., Hubbard et al., 2010; Marsee y Frick, 2006). Aun teniendo en cuenta la necesidad de profundizar en este tipo relaciones, se podría plantear que quizás desde el ámbito familiar la presencia de cualquier tipo de alteración conductual haga considerar a los progenitores la posibilidad de que sus hijos presenten problemas a cualquier nivel, sin llegar a distinguir de forma clara entre la dimensión externalizante e internalizante de la conducta.

Por otra parte, la manifestación temprana de rasgos psicopáticos, especialmente de tipo conductual, se relaciona en líneas generales con ambos tipos de conductas agresivas. Sin embargo, de forma específica y en relación con las variables autoinformadas, se observan importantes niveles de asociación entre los rasgos conductuales centrados en la impulsividad y agresión reactiva, tradicionalmente definida por su carácter colérico e impulsivo (Dodge, 1991; Dodge y Coie, 1987). Únicamente en los informes de padres se observan mayores niveles de conductas agresivas proactivas entre los jóvenes que han comenzado a manifestar rasgos de dureza e insensibilidad emocional, un resultado reiteradamente corroborado en los estudios sobre personalidad psicopática infanto-juvenil la cual ha sido asociada con el desarrollo de conductas agresivas, especialmente de tipo proactivo (Marsee y Frick, 2010).

En cuanto a las restantes variables de personalidad analizadas, se ha observado una relación significativa entre agresión proactiva y extraversión, lo cual podría ir en la línea de los trabajos que apuntan que los jóvenes más proactivos poseen mayores habilidades sociales y suelen sufrir menor rechazo por parte de los iguales (Brendgen et al., 2001). Por su parte, la agresividad reactiva se relacionó con menores niveles de responsabilidad, un resultado probablemente asociado con los elevados niveles de impulsividad observados en este grupo (Hubbard et al., 2010).

Otras de las variables analizadas hacían referencia a las habilidades de competencia social. En este sentido, tal y como se esperaba, se comprobó que los menores niveles de habilidades sociales, de comunicación y de regulación emocional se manifestaban entre el grupo de jóvenes con mayor tendencia a la agresión impulsiva y reactiva, confirmando los déficits en dichas habilidades como uno de los correlatos que por definición configuran la agresión reactiva (Dodge y Coie, 1987; Hubbard et al., 2001; Marsee y Frick, 2007). 
En cuanto a las variables familiares, tanto las prácticas familiares coercitivas como los procesos familiares disfuncionales (interacciones paterno-filiales conflictivas y baja supervisión) se relacionaron con la manifestación de conductas agresivas de tipo reactivo, tal y como se observó en estudios anteriores (Hubbard et al., 2010). De este modo, parece que el empleo de un estilo parental autoritario, basado en la coerción y el castigo, así como la presencia de relaciones familiares empobrecidas podrá favorecer el desarrollo de conductas agresivas reactivas (Vitaro et al., 1998). Sin embargo, a pesar de que algunos autores señalaron la influencia familiar en el desarrollo de la agresividad proactiva (Vitaro et al., 2006), así como su posible papel mediador en la relación establecida entre agresión proactiva y delincuencia futura (Brendgen et al., 2001), a nivel transversal no se ha constatado una relación clara, siguiendo la línea de las formulaciones que plantean que las variables familiares y los procesos de socialización juegan un papel más relevante en el desarrollo de la agresión reactiva (Hubbard et al., 2010; Raine et al., 2006).

En definitiva, los resultados obtenidos en este trabajo permiten observar la existencia de relaciones diferenciales entre agresión reactiva-proactiva y diversas variables emocionales, conductuales, familiares y de personalidad. Estos resultados justifican la conceptualización de la conducta agresiva adolescente en base a la motivación que la impulsa (Kokko y Pulkkinen, 2005). De este modo, se sustenta la distinción entre agresión reactiva, relacionada con impulsividad, TDAH, problemas de internalización, menor competencia social (déficits en habilidades sociales, de comunicación y regulación emocional) y prácticas parentales disfuncionales, y agresión proactiva, relacionada con un peor ajuste escolar, problemas de conducta y bajos niveles de ansiedad (Andreu et al., 2006; Hubbard et al., 2010).

Con todo ello el trabajo presenta ciertas limitaciones que deberán ser subsanadas en investigaciones futuras. En primer lugar, se ha empleado una metodología correlacional que, a pesar de facilitar el conocimiento de los principales correlatos de la agresión adolescente justificando así su conceptualización, limita el análisis en profundidad de dichas relaciones diferenciales, sobre todo teniendo en cuenta el solapamiento existente y reiteradamente constatado entre agresión reactiva y proactiva así como las relaciones asimétricas presentadas entre ambos tipos de agresión (Crapanzano et al., 2010). Este solapamiento, aun tras ser controlado mediante correlaciones parciales, viola ciertos 
requisitos que deben ser asumidos en el empleo de las metodologías correlacionales en el análisis de correlatos y efectos diferenciales (Cohen, 1983) dificultando así la interpretación de resultados (Vitaro et al., 1998). Es por ello que en futuros planteamientos se deberán emplear métodos de control más exhaustivos (Marsee y Frick, 2006); del mismo modo, podrán ser abordados desde aproximaciones centradas en la persona (e.g., análisis cluster; Barker et al., 2006; Muñoz et al., 2008). Por otra parte, el presente trabajo únicamente ofrece una perspectiva transversal de las relaciones diferenciales entre agresión reactiva/proactiva y un amplio conjunto de variables emocionales, conductuales, familiares y de personalidad. Con el fin de poder dotar de direccionalidad a las relaciones halladas los futuros trabajos deberán ser diseñados desde una perspectiva longitudinal que permitirá desentrañar las causas subyacentes a la conducta agresiva adolescente, así como las relaciones predictivas que ambos tipos de agresión mantienen con conductas antisociales y delictivas futuras o las trayectorias evolutivas que desarrollarán con el paso de los años (Barker et al., 2006; Brendgen et al., 2001; Raine et al., 2006). Finalmente, teniendo en cuenta las diferencias observadas en la literatura previa entre chicos y chicas (e.g., Crapanzano et al., 2010) será necesario tener en cuenta la perspectiva de género en futuros trabajos.

Con estas limitaciones en mente, los resultados de este trabajo han permitido sustentar la conceptualización que distingue entre agresión reactiva y proactiva. Teniendo en cuenta la existencia de correlatos claramente diferenciales y la posibilidad, cada vez más asumida, de que ambos tipos de conductas agresivas desarrollen diversas trayectorias evolutivas relacionadas en mayor o menor medida con problemas de conducta severos y persistentes (Raine et al., 2006), estos resultados justifican el empleo de estrategias de intervención diferenciadas en función de la manifestación de uno $u$ otro tipo de agresión (Marsee y Frick, 2010). De este modo, la agresión de tipo reactivo podrá ser abordada a partir de estrategias centradas en el control de la impulsividad, la ansiedad o el desarrollo de habilidades sociales y regulación emocional, al tiempo que la agresión proactiva se podrá manejar a partir del trabajo específico de las creencias y percepciones positivas acerca del uso de la agresión como forma de obtener aquello que se desea, así como el abordaje a nivel emocional de las carencias en empatía y consideración hacia los demás (Crapanzano et al., 2010; Marsee y Frick, 2007). 


\section{Referencias}

Achenbach, T. M. (1991a). Manual for the Child Behavior Checklist and 1991 Profile. Burlington: University of Vermont.

Achenbach, T. M. (1991b). Manual for the Teacher's Report Form and 1991 Profile. Burlington: University of Vermont.

Andreu, J.M., Ramírez, J.M. y Raine, A. (2006). Un modelo dicotómico de la agresión: Valoración mediante dos autoinformes (CAMA y RPQ). Psicopatología Clínica, Legal y Forense, 5, 25-42.

Barker, E. D., Tremblay, R. E., Nagin, D. S., Vitaro, F. y Lacourse, E. (2006). Development of male proactive and reactive physical aggression during adolescence. Journal of Child Psychology and Psychiatry, 47, 783-790.

Berkowitz, L. (1993). Aggression: Its causes, consequences, and control. Nueva York: Academic.

Berry, J.W., Phinney, J.S., Sam, D. and Vedder, P. (Eds.) (2006). Immigrant youth in cultural transition: Acculturation, identity and adaptation across national contexts. Hillsdale: Erlbaum.

Brendgen, M., Vitaro, F., Tremblay, R. E., y Lavoie, F. (2001). Reactive and proactive aggression: Predictions to physical violence in different contexts and moderating effects of parental monitoring and caregiving behavior. Journal of Abnormal Child Psychology, 29, 293-304.

Bryant, B. (1982). An index of empathy for children and adolescents. Child Development, 53, 413-425.

Bushman, B. J., y Anderson, C. A. (2001). Is it time to pull the plug on hostile versus instrumental aggression dichotomy? Psychological Review, 108, 273-279.

Cohen, J. (1983). The cost of dichotomization. Applied Psychological Measurement, 7, 249-253.

Cohen, R., Hsueh, Y., Russell, K. M. y Ray G. E. (2006). Beyond the individual: a consideration of context for the development of aggression. Aggression and Violent Behavior, 11, 341-351.

Conduct Problems Prevention Research Group (1995). Social Competence Scale (Parent Version). University Park, PA: Pennsylvania State University.

Conners, C.K. (1997). Conners Rating Scales-Revised. Toronto: Multi-Health System. 
Crapanzano, A. M., Frick, P. J. y Terranova, A. M. (2010). Patterns of physical and relational aggression in a school-based sample of boys and girls. Journal of Abnormal Child Psychology, 38, 433-445.

Cronbach, L. J. (1951). Coefficient alpha and the internal structure of tests. Psychometrika, 16, 297-334.

Dodge, K. A. (1991). Emotion and social information processing. En J. Garber y K.A. Dodge (Eds.). The development of emotion regulation and dysregulation (pp. 159181). Nueva York: Cambridge University Press.

Dodge, K. A. y Coie, J. D. (1987). Social information processing factors in reactive and proactive aggression in children's peer groups. Journal of Personality and Social Psychology, 53, 1146-1158.

Dodge, K. A., Lochman, J. E., Harnish, J. D., Bates, J. E. y Pettit, G. S. (1997). Reactive and proactive aggression in school children and psychiatrically impaired chronically assaultive youth. Journal of Abnormal Psychology, 106, 37-51.

Donnellan, M.B., Oswald, F.L., Bairs, B.M. y Lucas, R.E. (2006). The Mini-IPIP Scales: Tiny-yet-effective measures of the Big Five Factors of personality. Psychological Assessment, 18, 192-203.

Eysenck, S. B., Easting, G. y Pearson, P. (1984). Age norms for impulsiveness, venturesomeness and empathy in children. Personality and Individual Differences, 5, 315-321.

Frick, P. J., Cornell, A., Bodin, S., Dane, H., Barry, C. y Loney, B. (2003). Callousunemotional traits and developmental pathways to severe conduct problems. Developmental Psychology, 39, 246-260.

Frick, P. J. y Hare, R. D. (2001). The Antisocial Process Screening Device. Toronto: Multi-Health System.

Frick, P. J. y Marsee, M. A. (2006). Psychopathy and developmental pathways to antisocial behavior in youth. En C.J. Patrick (Ed.), Handbook of Psychopathy (pp. 353-374). Nueva York: Guilford.

Frick, P.J., O’Brien, BS., Wootton, J.M. y McBurnett, K. (1994). Psychopathy and conduct problems in children. Journal of Abnormal Psychology, 103, 54-58.

Goldberg, L.R. (1999). A broad-bandwidth, public domain, personality inventory measuring the lower level facets of several five-factor models. En I. Mervielde, I. 
Deary, F. De Fruyt y F. Ostendorf (Eds.), Personality Psychology in Europe (Vol. 7, pp. 7-82). Tilburg, Holland: Tilburg University Press.

Hubbard, J. A., Dodge, K. A., Cillessen, A. H. N., Coie, J. D. y Schwartz, D. (2001). The dyadic nature of social information processing in boys reactive and proactive aggression. Journal of Personality and Social Psychology, 80, 268-280.

Hubbard, J. A., McAuliffe, M. D., Morrow, M. T. y Romano, L. J. (2010). Reactive and proactive aggression in childhood and adolescence: Precursors, outcomes, processes, experiences and measurement. Journal of Personality, 78, 95-118.

Hubbard, J. A., Smithmyer, C. M., Ramsden, S. R., Parker, E. H., Flanagan, K. D., Dearing, K. F. et al., (2002). Observational, physiological, and self-report measures of children`s anger: Relations to reactive versus proactive aggression. Child Development, 73, 1101-1118.

Kokko, K. y Pulkkinen, L. (2005). Stability of aggressive behavior from childhood to middle age in women and men. Aggressive behavior, 31, 485-497.

Little, T. D., Jones, S. M., Henrich, C. C. y Hawley, P. H. (2003). Disentangling the "whys" from "whats" of aggressive behavior. International Journal of Behavioral Development, 27, 122-133.

López-Romero, L. y Romero, E. (2010). Goals during adolescence and their relationship with antisocial behavior. The Spanish Journal of Psychology, 13, 166177.

López-Romero, L., Romero, E. y Luengo, M.A. (en prensa). La personalidad psicopática como indicador distintivo de severidad y persistencia en los problemas de conducta infanto-juveniles. Psicothema.

Marsee, M. A. y Frick, P. J. (2007). Exploring the congntive and emotional correlates to proactive and reactive aggression in a sample of detained girls. Journal of Abnormal Child Psychology, 35, 969-981.

Marsee, M. A. y Frick, P. J. (2010). Callous unemotional traits and aggression in youth. En A. William y L. Elizabeth (Eds.), Emotions, aggression and morality in children: Bridging development and psychopathology (pp. 137-156). Washington: American Psychological Association.

Molina, A. (2001). Instrumentos de evaluación clínica en niños y adolescentes. Revista de Psiquiatría y Psicología del Niño y el Adolescente, 2, 23-40. 
Muñoz, L. C., Frick, P. J., Kimonis, E. R. y Aucoin, K. J. (2008). Types of aggression, responsiveness to provocation and callous-unemotional traits in detained adolescents. Journal of Abnormal Child Psychology, 36, 15-28.

Musitu, G. y García, F. (2001). Escala de Socialización Parental en la Adolescencia. Madrid: TEA.

Raine, A., Dodge, K., Loeber, R., Gatze-Kopp, L., Lynam, D., Reynolds, C. et al., (2006). The Reactive-Proactive Aggression Questionnaire: Differential correlates of reactive and proactive aggression in adolescent boys. Aggressive Behavior, 32, 159171.

Russo, M. F., Stokes, G. S., Lahey, B. B., Christ, M. A. G., McBurnett, K., Loeber, R. et al. (1993). A sensation-seeking scale for children: A further refinement and psychometric development. Journal of Psychopathology and Behavioral Assessment, 15, 69-86.

Silva, F., Martorell, C. y Clemente, A. (1986). Socialization and personality: Study through questionnaires in a preadult Spanish population. Personality and Individual Differences, 7, 355-372.

Seisdedos, N. (1982). Cuestionarios de Ansiedad Estado-Rasgo. Madrid: TEA.

Spielberg, C.D., Gorsuch, R.L. y Lushene, R. (1970). Manual of the State/Trait Anxiety Inventory. Palo Alto, CA: Consulting Psychologist Press.

Vazsonyi, A.T., Hibbert, J.R. y Snider, J.B. (2003). Exotic enterprise no more? Adolescent reports of family and parenting processes form youth in four countries. Journal of Research on Adolescence, 13, 129-160.

Vitaro, F., Brendgen, M. y Barker, E.D. (2006). Subtypes of aggressive behaviors: A developmental perspective. International Journal of Behavioral Development, 30, 12-19.

Vitaro, F., Brendgen, M. y Tremblay, R. E. (2002). Reactively and proactively aggressive children: Antecedent and subsequent characteristics. Journal of Child Psychology and Psychiatry and Allied Disciplines, 43, 495-506.

Vitaro, F., Gendreau, P. L., Tremblay, R. E. y Oligny, P. (1998). Reactive and proactive aggression differentially predict later conduct problems. Journal of Child Psychology and Psychiatry, 39, 377-385.

Walters, G.D. (2005). Proactive and reactive aggression: a lifestyle view. En J.P. Morgan (Ed.), Psychology of Aggression (pp. 29-43). Hauppauge: Nova Science. 
White, S. T. y Frick, P. J. (2010). Callous-unemotional traits and their importance to causal models of severe antisocial behavior in youth. En R. T. Salekin y D. T. Lynam (Eds.), Handbook of Child and Adolescent Psychopathy (pp. 135-156). Nueva York: The Guilford Press. 